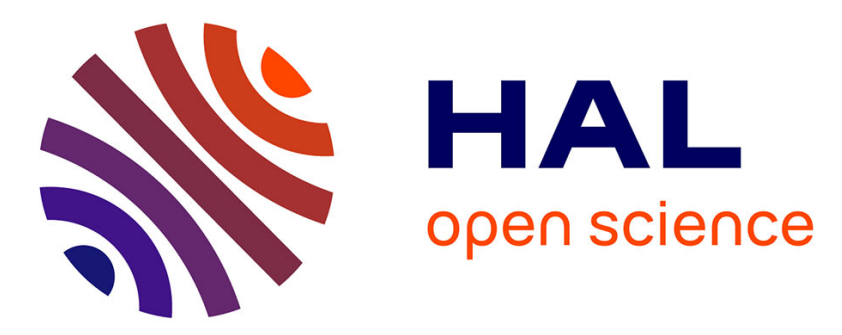

\title{
Reservoir simulation model calibration methodology with polymer flooding based on laboratory experiments
}

\author{
C. Preux, I. Malinouskaya
}

\section{To cite this version:}

C. Preux, I. Malinouskaya. Reservoir simulation model calibration methodology with polymer flooding based on laboratory experiments. Journal of Petroleum Science and Engineering, 2019, 181, pp.106176. 10.1016/j.petrol.2019.06.040 . hal-02276277

\section{HAL Id: hal-02276277 \\ https://hal-ifp.archives-ouvertes.fr/hal-02276277}

Submitted on 2 Sep 2019

HAL is a multi-disciplinary open access archive for the deposit and dissemination of scientific research documents, whether they are published or not. The documents may come from teaching and research institutions in France or abroad, or from public or private research centers.
L'archive ouverte pluridisciplinaire HAL, est destinée au dépôt et à la diffusion de documents scientifiques de niveau recherche, publiés ou non, émanant des établissements d'enseignement et de recherche français ou étrangers, des laboratoires publics ou privés. 


\title{
Reservoir simulation model calibration methodology with polymer flooding based on laboratory experiments
}

\author{
C. Preux ${ }^{\mathrm{a}}$, I. Malinouskaya ${ }^{\mathrm{a}}$ \\ ${ }^{a}$ IFP Energies Nouvelles \\ 1 et 4 avenue de Bois-Préau, 92852 Rueil-Malmaison Cedex, France
}

\begin{abstract}
Due to its high apparent viscosity, polymer is considered as an alternative to water as injection fluid in hydrocarbon reservoirs. The mobility of the waterpolymer mixture is much smaller than that of water alone, which helps prevent undesirable viscous fingering. Nevertheless, the selection of a polymer process involves a procedure for assessing the performance of the polymer in terms of mobility reduction. This procedure involves laboratory experiments in which the pressure losses are measured during polymer displacement, as well as during water displacement. In the next step of the procedure, a model is calibrated by simulation in order to predict the efficiency of the polymer. Although the polymer models traditionally used at this stage are relatively simple, their calibration from the results of the laboratory tests is much more intricate, insofar as the mobility reduction generated by the polymer depends not only on the concentration but also on the temperature, the salinity and the shear rate. In this paper, we put forward a new approach to this multivariate problem and propose a rigorous calibration methodology which allows for a fast physical representativity, thus limiting the number of laboratory experiments. This methodology is the subject of a pending patent application (FR 18/58547).
\end{abstract}

Keywords: polymer flooding, simulation, mobility reduction

\section{Introduction}

In order to improve the oilfield recovery factor, many oil companies resort to adding polymer in injected water. The idea is to take advantage of the large viscosity of the polymer in order to reduce the mobility of the waterpolymer mixture. Numerous experimental studies show that the mobility 
of the latter strongly depends on various parameters such as the polymer concentration $c$, the shear rate due to the polymer flooding velocity $\dot{\gamma}$, the temperature $T$ and the salinity or ionic strength $I_{\mu}$ in case of salted water [15], [17], [19]. For example, a high impact of the low salinity on the polymer behavior, in particular, on the mobility reduction, was demonstrated by [2]. In [21], the study of the slug-based compositionally-tuned polymer flooding reveals that the polymer viscosity can be controlled through the mixture salinity. In most theoretical studies, summarized by [20], the analyses are carried out only for one or two parameters at a time. For example, in [1], the authors use calibrated model to consider polymer concentration and salinity effect, but neglect the temperature effects.

For the calibration of the reservoir simulation model to take into account all of the mentioned physical effects, we have to conduct a large number of experiments corresponding to all possible combinations of the chosen parameters $\left\{c, \dot{\gamma}, T, I_{\mu}\right\}$. This turns out to be expensive and impractical to implement.

The present work addresses this calibration issue by introducing a methodology that requires as few laboratory experiments as possible. This methodology enables us to obtain a complete description of the mobility reduction as a function of any set of parameters $\left\{c, \dot{\gamma}, T, I_{\mu}\right\}$. After describing a general polymer model in Section 2, we provide full details of the calibration methodology in Section 3. Step by step, the dependencies on the polymer concentration, the salinity/ionic force, the shear rate and the temperature are taken into account and the required measurements associated with these parameters are stated. Then, in Section 4, we apply the methodology to an experimental data set $[15,17]$ and compute the corresponding mobility reduction function. Finally, we draw some conclusions on the efficiency of this methodology and discuss some prospects of the present work in Section 5 .

\section{Polymer model formulation}

The polymer displacement model is based on the assumption that the polymer-added water has a reduced mobility. The derivation of this model, used by many reservoir simulators, relies on a modification of the water viscosity which is expressed as

$$
\mu_{w p}=R_{m}\left(c, I_{\mu}, \dot{\gamma}, T\right) \mu_{w}
$$


where $\mu_{w}$ is the viscosity of the injection water, $\mu_{w p}$ the viscosity of the water containing polymer and $R_{m}\left(c, T, I_{\mu}, \dot{\gamma}\right)$ the mobility reduction. The latter is a function of the polymer concentration $c$, the temperature $T$, the salinity/ionic strength $I_{\mu}$ and the shear rate $\dot{\gamma}$. The determination of the mobility reduction function $R_{m}\left(c, I_{\mu}, \dot{\gamma}, T\right)$ from experimental data is the object of model calibration for the purpose of predicting polymer displacements. Notice that the total mobility reduction from polymer injection is caused by two effects:

- the increase in polymer viscosity,

- the reduction in permeability presumably by polymer retention, adsorption, and inaccessible pore volume.

In this paper, we consider only the increase in polymer viscosity. The permeability reduction $R_{k}$, which appears in the phase velocity such as

$$
\vec{u}_{w}=-\frac{K k_{r}}{R_{k} R_{m} \mu_{w}}\left(\vec{\nabla} p_{w}+\rho_{w} g\right)
$$

is not studied in this paper.

In practice, the laboratory measurements allow us to obtain the solvent viscosity $\mu_{w p}$ and to deduce the corresponding value of $R_{m}$ as a relative viscosity $\mu_{w p} / \mu_{w}$. On the other hand, these results can be considered in terms of the reduced specific viscosity $\left(\mu_{w p}-\mu_{w}\right) /\left(c \mu_{w}\right)$, where $\mu_{w}$ is the water viscosity. This gives rise to the intrinsic viscosity

$$
[\mu]=\lim _{c \rightarrow 0} \frac{\mu_{w p}-\mu_{w}}{\mu_{w} c} .
$$

The well-known formulation for the polymer concentration dependence proposed by Huggins [10] is written as

$$
R_{m}(c)=1+[\mu] c+k^{\prime}([\mu] c)^{2},
$$

where $[\mu]$ is defined by (3) and $k^{\prime}$ is the Huggins coefficient. This formula is treating bulk polymer solutions but is used by default in different models of polymer flooding $([24,18,13,9])$. The intrinsic viscosity of the polymer mixture $[\mu]$ characterizes its so-called viscosifying power. This quantity represents the expansion volume of the polymer in a water of a given salinity at a given temperature and a shear rate, and is expressed in $\mathrm{cm}^{3} / \mathrm{g}$ or in $\mathrm{L} / \mathrm{g}$. 


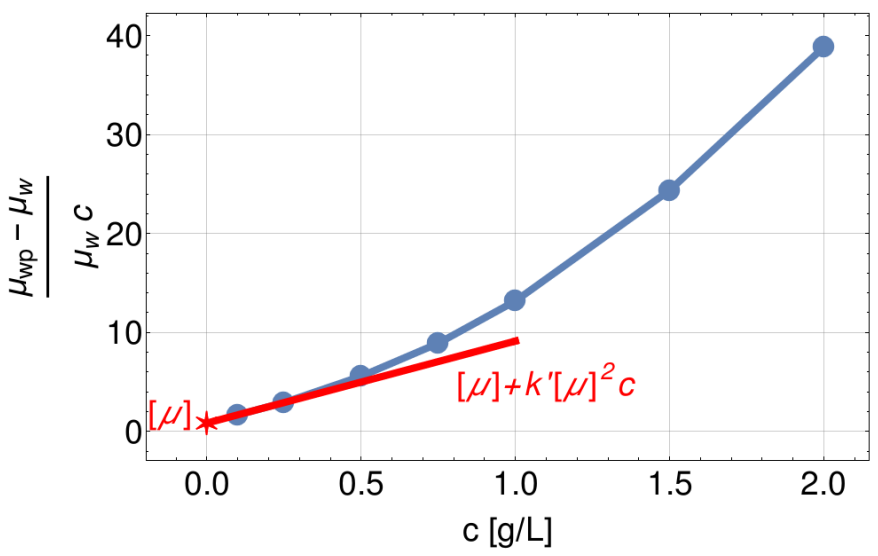

Figure 1: Reduced specific viscosity function of polymer concentration. (Reduced specific viscosity in blue and linear part of the specific viscosity in red.)

The Huggins coefficient $k^{\prime}$ is a dimensionless number; for the hydrolyzed polyacrylamide polymers (HPAM) in a good solvent, its value is about $0.4 \pm 0.1$ [15].

Using definition (3) and formulation (4), $[\mu]$ and $k^{\prime}$ result from the best fit of a linear part of the specific viscosity (reduced to low concentration values) as shown in Figure 1. The dependences on the temperature, the salinity and the shear rate will be managed through these two parameters. Finally, we search a formulation based on these dependances:

$$
R_{m}\left(c, I_{\mu}, \dot{\gamma}, T\right)=1+[\mu]\left(I_{\mu}, \dot{\gamma}, T\right) c+k^{\prime}\left(I_{\mu}, T\right)\left([\mu]\left(I_{\mu}, \dot{\gamma}, T\right) c\right)^{2}
$$

The principal assumption in this methodology is that the different parameters $\left(c, I_{\mu}, \dot{\gamma}, T\right)$ are constant with respect to others (salt, shear rate and temperature). We search also the intrinsic viscosity and the Huggins coefficient in an uncoupling form:

$$
\begin{aligned}
{[\mu]\left(I_{\mu}, \dot{\gamma}, T\right) } & =[\mu]_{I_{\mu}}\left(I_{\mu}\right) \cdot[\mu]_{\dot{\gamma}}(\dot{\gamma}) \cdot[\mu]_{T}(T) \\
k^{\prime}\left(I_{\mu}, T\right) & =k_{I_{\mu}}^{\prime}\left(I_{\mu}\right) \cdot k_{T}^{\prime}(T)
\end{aligned}
$$

It remains a simplifying assumption to limit the number of experimental data and to limit the variables and we show in the last section that this assumption gives a correct match. 


\section{Calibration methodology of the mobility reduction function}

In order to calibrate the polymer model described above, we can use polymeric water displacements results, obtained under given displacement conditions (polymer concentration, injection rates, temperature and salinity). Usually, these displacements are carried out on cores initially saturated with a liquid phase (water and/or oil), which is mobile or residual depending on the core history and the purpose of the measurements. The considered displacements are the drainage processes with the increasing water saturation.

For each displacement, the laboratory must provide the following measurements:

- the evolution of the pressure drop along the core: the ratio between the two stabilized pressure losses measured at the end of each displacement is the main result of a couple of displacements and is called mobility reduction;

- the liquid phase (water and/or oil) and gas productions.

Less accurate but faster, we can determine rheological properties of polymer by viscosimeter. In this case we treat only bulk solution polymer viscosity without taking into account the porous media effect. We obtain finally a correlation which can be considered as a starting point for the coreflood history match.

The calibration of the mobility reduction of a given rock-fluids-additive system involves the displacement measurements for any possible combination of parameters $\left\{c, I_{\mu}, \dot{\gamma}, T\right\}$.

As shown before, the dependence on the concentration is expressed by (4) and the dependence on the ionic strength $I_{\mu}$, the shear rate $\dot{\gamma}$ and the temperature $T$ is contained in the intrinsic viscosity $[\mu]$ and the Huggins coefficient $k^{\prime}$. Therefore, the calibration methodology consists of the following steps. First, we choose a reference set of parameters $\left\{I_{\mu, 1}, \dot{\gamma}_{1}, T_{1}\right\}$ and use them for the first series of measurements with various polymer concentrations $c_{i}(i=1, \ldots, N)$ to deduce the intrinsic viscosity and Huggins coefficient as described before (Figure 1). Schematically,

$$
S 1:\left\{\left\{c_{i}\right\}_{i=1}^{N}, I_{\mu, 1}, \dot{\gamma}_{1}, T_{1}\right\} \rightarrow\left\{[\mu]_{1}, k_{1}^{\prime}\right\} .
$$

It should be noted that for further application of the methodology, it is convenient to restrict the shear rate $\dot{\gamma}_{1}$ to very small values, namely, $\dot{\gamma}_{1} \rightarrow 0$. 
Then, in order to study the influence of the ionic strength, we modify the reference value of $I_{\mu}$. We perform the same series of measurements as before to deduce the corresponding values of $[\mu]\left(I_{\mu}\right)$ and $k^{\prime}\left(I_{\mu}\right)$. This procedure can be repeated several times to obtain the curves of the intrinsic viscosity and Huggins coefficient as functions of $I_{\mu}$. However, we will demonstrate that our methodology only needs one additional series of experiments with a new value of the ionic strength.

Similarly, changing the shear rate and the temperature in the reference set of parameters, we get the corresponding intrinsic viscosities $[\mu](\dot{\gamma})$ and $[\mu](T)$ and Huggins coefficients $k^{\prime}(\dot{\gamma})$ and $k^{\prime}(T)$, respectively. Again, we will demonstrate that only one additional series of measurements per parameter is required to obtain a full description of the intrinsic viscosity as a function of this parameter. Nevertheless, the dependence of the Huggins coefficient on the temperature in presence of a variable salinity appears to be more complex and requires one additional series of measurements with simultaneously modified values of $I_{\mu}$ and $T$.

\subsection{Determination of salinity/ionic strength dependence}

We consider here the dependence on the ionic strength $I_{\mu}$, keeping in mind that the dependence on the salinity $s$ can be determined exactly in the same way since $I_{\mu}$ depends on the salinity $s=T D S$ (for Total Dissolved Salts). For example, considering 3 pseudo-ions, we use modified ionic strength which can be estimated according to the composition of the salt as

$$
I_{\mu}(s)=\frac{C_{+}}{M_{+}}+\alpha_{\mu} \frac{C_{++}}{M_{++}}+\beta_{\mu}\left(\frac{C_{++}}{M_{++}}\right)^{2},
$$

where the molar masses $M_{+,++,-}$and the mass concentrations $C_{+,++,-}$correspond to the 3 groups of pseudo-ions: 2 pseudo-cations $\mathrm{I}+$, I++ and 1 pseudoanion I-. This formula (9) is based on the reservoir simulator $\sum C O R E P O L$ presented in [11] and [12]. Then, this modified ionic strength was adopted in the reservoir simulator $S A R I P^{C H}$ and PumaFlow ${ }^{T M}$. It take into account the fact that divalent ions have a strong influence on polymer viscosity. In [7], the author show that the effect of divalent ions is observed by a significant reduction in the viscosity of the polymer solution. It seems that the divalent ions are tightly bind to anions along the polyelectrolyte chain because it has a higher charge, causing the polymer chain to contract to its minimum size. For polymer type HPAM, the reservoir simulator $\sum C O R E P O L$ adviced to 
choose $\alpha_{\mu}=10.5$ and $\beta_{\mu}=216$. The intrinsic viscosity is prescribed as a linear function of $I_{\mu}^{-1 / 2}[14,19]$, that is,

$$
[\mu]\left(I_{\mu}\right)=\left[\mu_{0}\right]+b_{I} I_{\mu}^{-1 / 2},
$$

where $\left[\mu_{0}\right]$ is the intrinsic viscosity when $I_{\mu} \rightarrow \infty$. There are two unknown parameters $\left[\mu_{0}\right]$ and $b_{I}$. Therefore, one additional series of measurements is required. For this, we use the reference set S1 (8), set an ionic strength to $I_{\mu, 2}$ and proceed to a new series of measurements

$$
S 2:\left\{\left\{c_{i}\right\}_{i=1}^{N}, I_{\mu, 2}, \dot{\gamma}_{1}, T_{1}\right\} \rightarrow\left\{[\mu]_{2}, k_{2}^{\prime}\right\}
$$

The results of S1 and S2 are inserted into formulation (10) and yield a system of two linear equations in two unknowns. The solution of this system provides the parameters $\left[\mu_{0}\right]$ and $b_{I}$ as

$$
\begin{aligned}
b_{I} & =\frac{[\mu]_{1}-[\mu]_{2}}{I_{\mu, 1}^{-1 / 2}-I_{\mu, 2}^{-1 / 2}} \\
{\left[\mu_{0}\right] } & =[\mu]_{2}-\frac{[\mu]_{1}-[\mu]_{2}}{I_{\mu, 1}^{-1 / 2}-I_{\mu, 2}^{-1 / 2}} I_{\mu, 2}^{-1 / 2}
\end{aligned}
$$

Thus, the intrinsic viscosity as a function of the ionic strength is obtained by

$$
[\mu]\left(I_{\mu}\right)=[\mu]_{2}+\frac{[\mu]_{1}-[\mu]_{2}}{I_{\mu, 1}^{-1 / 2}-I_{\mu, 2}^{-1 / 2}}\left(I_{\mu}^{-1 / 2}-I_{\mu, 2}^{-1 / 2}\right) .
$$

According to [23], the Huggins coefficient depends on the salt composition and the concentration range. The lineary dependance seems better adapted to weak ionic forces. For high ionic strengths, however, there is too few experimental results to be more affirmative. In this paper, at first approximation, we decide to consider the Huggins coefficient as a linear function of $I_{\mu}$

$$
k^{\prime}\left(I_{\mu}\right)=\alpha_{I} I_{\mu}+\beta_{I}
$$

Similarly to the intrinsic viscosity, the results of the two sets of measurements $\mathrm{S} 1$ and $\mathrm{S} 2$ provide the parameters $\alpha_{I}$ and $\beta_{I}$ in the form

$$
\begin{aligned}
\alpha_{I} & =\frac{k_{1}^{\prime}-k_{2}^{\prime}}{I_{\mu, 1}-I_{\mu, 2}}, \\
\beta_{I} & =k_{2}^{\prime}-\frac{k_{1}^{\prime}-k_{2}^{\prime}}{I_{\mu, 1}-I_{\mu, 2}} I_{\mu, 2} .
\end{aligned}
$$


The definition of the intrinsic viscosity $[\mu]\left(I_{\mu}\right)$ and the Huggins coefficient $k^{\prime}\left(I_{\mu}\right)$ through analytical formulas involving two parameters $\left(\left[\mu_{0}\right]\right.$ and $b_{I}$ for viscosity and $\alpha_{I}$ and $\beta_{I}$ for $k^{\prime}$ ) implies two series of experiments corresponding to the two different values of $I_{\mu}$ for any ionic strength, but for the same conditions on the temperature and shear rate.

\subsection{Determination of shear rate dependence}

Very often the power law is used to fit the viscosity measurements as a function of the shear rate in the case of non-Newtonian fluids $[14,15]$. The Cross model [6] was found more efficient for a wide range of the shear rates as well as in Newtonian fluids at small shear rate. The most generalized model proposed in the literature is the Carreau model [4], which reads

$$
\frac{\mu(\dot{\gamma})-\mu_{\dot{\gamma} \infty}}{\mu_{\dot{\gamma} 0}-\mu_{\dot{\gamma} \infty}}=\left[1+(\lambda \dot{\gamma})^{2}\right]^{(n-1) / 2}
$$

where $\mu_{\dot{\gamma} \infty}$ and $\mu_{\dot{\gamma} 0}$ are the Newtonian viscosities corresponding to $\dot{\gamma} \rightarrow \infty$ and $\dot{\gamma} \rightarrow 0$, respectively; here, $\lambda$ is a time constant that corresponds to a characteristic shear rate, i.e.,

$$
\lambda=\left(\dot{\gamma}_{c}\right)^{-1},
$$

where $\dot{\gamma}_{c}$ depends on the type of media. For example, in [5], it is defined as

$$
\dot{\gamma}_{c}=a \frac{4 u}{r_{h}}
$$

where $a$ is a form factor that can be obtained experimentally, $u=Q / \phi S$ with

$Q$ the flow rate, $S$ the section area and $\phi$ the porosity, and $r_{h}$ is a specific pore size $r_{h}=\sqrt{8 K / \phi}$ with $K$ the permeability.

The viscosity $\mu_{\dot{\gamma} \infty}$ can be set to the viscosity of the solvent without polymer [15]. Therefore, the Carreau model can be written in terms of intrinsic viscosity as

$$
[\mu](\dot{\gamma})=\frac{\left[\mu_{\dot{\gamma} 0}\right]}{\left[1+\left(\frac{\dot{\gamma}}{\dot{\gamma}_{c}}\right)^{2}\right]^{(1-n) / 2}} .
$$

Since the imposed shear rate in the reference set of parameters S1 (8) is very small, we can write

$$
\left[\mu_{\dot{\gamma} 0}\right]=[\mu]\left(I_{\mu}\right),
$$


where $[\mu]\left(I_{\mu}\right)$ is obtained by $(14)$.

A series of measurements, say S3, with a new value of the shear rate, $\dot{\gamma}_{2}$, is required in order to determine the parameter $n$ in (20). In other words,

$$
S 3:\left\{\left\{c_{i}\right\}_{i=1}^{N}, I_{\mu, 1}, \dot{\gamma}_{2}, T_{1}\right\} \rightarrow\left\{[\mu]_{3}, k_{3}^{\prime}\right\} .
$$

Similarly to the previous case, the results of S1 and S3 are plugged into formulation (20) and lead to a system of equations from which we obtain $n$ under the form

$$
n=1-\frac{2 \log \left(\frac{[\mu]_{1}}{[\mu]_{3}}\right)}{\log \left(1+\left[\frac{\dot{\gamma}_{2}}{\dot{\gamma}_{c}}\right]^{2}\right)} .
$$

Then, the intrinsic viscosity as a function of the shear rate and the ionic strength is inferred as

$$
[\mu]\left(I_{\mu}, \dot{\gamma}\right)=\frac{[\mu]\left(I_{\mu}\right)}{\left[1+\left(\frac{\dot{\gamma}}{\dot{\gamma}_{c}}\right)^{2}\right]^{\frac{\log \left(\frac{[\mu]_{1}}{\mu]_{3}}\right)}{\log \left(1+\left[\frac{\dot{\gamma}_{2}}{\dot{\gamma}_{c}}\right]^{2}\right)}}} .
$$

Let us remind that (21) has been obtained under condition $\dot{\gamma} \rightarrow 0$. However, it can be generalized for any value of $\dot{\gamma}$ as

$$
\left[\mu_{\dot{\gamma} 0}\right]=[\mu]\left(I_{\mu}\right)\left[1+\left(\frac{\dot{\gamma}_{1}}{\dot{\gamma}_{c}}\right)^{2}\right]^{(1-n) / 2},
$$

with the same value of $n$ as found by (23). Therefore, the generalized formulation will differ from (24) by multiplier $\left[1+\left(\dot{\gamma}_{1} / \dot{\gamma}_{c}\right)^{2}\right]^{(1-n) / 2}$. The Huggins coefficient as a function of shear rate is generally defined as a constant.

\subsection{Determination of temperature dependence}

The intrinsic viscosity dependence on the temperature can be expressed under the form

$$
[\mu](T)=\left[\mu_{T_{0}}\right] M^{\alpha\left(\frac{1}{T}-\frac{1}{T_{0}}\right),}
$$

such as proposed by [3]. In (26), $\left[\mu_{T_{0}}\right]$ is the intrinsic viscosity at reference temperature $T_{0}, M$ is the molecular weight and $\alpha$ is a constant. Setting the reference temperature $T_{0}=T_{1}$, the intrinsic viscosity $\left[\mu_{T_{0}}\right]$ is determined as a function of the ionic strength and the shear rate obtained by (24), that is,

$$
\left[\mu_{T_{0}}\right]=[\mu]\left(I_{\mu}, \dot{\gamma}\right) .
$$


Then, a new series of measurements is required to deduce the second parameter $\alpha$. These measurements are performed using the reference set of parameters and setting a new temperature $T_{2}$. In a manner similar to the previous ones, by varying the polymer concentration $c$ we are in a position to obtain the intrinsic viscosity and the Huggins coefficient for this new set of parameters

$$
S 4:\left\{\left\{c_{i}\right\}_{i=1}^{N}, I_{\mu, 1}, \dot{\gamma}_{1}, T_{2}\right\} \rightarrow\left\{[\mu]_{4}, k_{4}^{\prime}\right\} .
$$

Solving the system of equations obtained substituting (8) and (28) into the formulation (26), we end up with the coefficient

$$
\alpha=\frac{\log \left(\frac{[\mu]_{1}}{[\mu]_{4}}\right)}{\log M}\left(\frac{1}{T_{1}}-\frac{1}{T_{2}}\right)^{-1} .
$$

Consequently, the intrinsic viscosity $[\mu]$ is expressed as a function of the ionic strength, the shear rate and the temperature as

$$
[\mu]\left(I_{\mu}, \dot{\gamma}, T\right)=[\mu]\left(I_{\mu}, \dot{\gamma}\right)\left(\frac{[\mu]_{1}}{[\mu]_{4}}\right)^{-\frac{T_{2}\left(T-T_{1}\right)}{T\left(T_{2}-T_{1}\right)}},
$$

where $[\mu]\left(I_{\mu}, \dot{\gamma}\right)$ was obtained by $(24)$.

The Huggins coefficient dependence on the temperature is imposed as a linear function

$$
k^{\prime}(T)=\alpha_{T} T+\beta_{T} .
$$

Similarly to the ionic strength dependence, two series of measurements, S1 and $\mathbf{S} 4$, are sufficient to determine the parameters $\alpha_{T}$ and $\beta_{T}$. However, they are found only for a given value of the ionic strength, $I_{\mu, 1}$. Taking into account the dependence on $I_{\mu}$ indicated by (13), we can turn the Huggins coefficient into the form

$$
k^{\prime}=\left(\alpha_{1} I_{\mu}+\alpha_{2}\right) T+\left(\beta_{1} I_{\mu}+\beta_{2}\right) .
$$

These cross-dependence on the ionic strength and the temperature implies a system of four equations in four parameters, while the measurements S1, S2 and S4 yield only three equations. Thus, one additional series of measurements is required with the ionic strength $I_{\mu, 2}$ (as in series S2) and the temperature $T_{2}$ (as in series S4), namely,

$$
S 5:\left\{\left\{c_{i}\right\}_{i=1}^{N}, I_{\mu, 2}, \dot{\gamma}_{1}, T_{2}\right\} \rightarrow\left\{[\mu]_{5}, k_{5}^{\prime}\right\} .
$$


Solving (31) for measurements S1 and S4, we obtain the two parameters $\alpha_{T}=\alpha_{T, 1}$ and $\beta_{T}=\beta_{T, 1}$ as

$$
\begin{aligned}
\alpha_{T, 1} & =\frac{k_{1}^{\prime}-k_{4}^{\prime}}{T_{1}-T_{2}} \\
\beta_{T, 1} & =k_{4}^{\prime}-\frac{k_{1}^{\prime}-k_{4}^{\prime}}{T_{1}-T_{2}} T_{2} .
\end{aligned}
$$

Similarly, for S2 and S5, the parameters in (31) are $\alpha_{T}=\alpha_{T, 2}$ and $\beta_{T}=\beta_{T, 2}$ and they are obtained by

$$
\begin{aligned}
\alpha_{T, 2} & =\frac{k_{2}^{\prime}-k_{5}^{\prime}}{T_{1}-T_{2}}, \\
\beta_{T, 2} & =k_{5}^{\prime}-\frac{k_{2}^{\prime}-k_{5}^{\prime}}{T_{1}-T_{2}} T_{2} .
\end{aligned}
$$

Therefore, it follows from (31)-(32) a system of four equations in the four parameters $\alpha_{1}, \alpha_{2}, \beta_{1}$ and $\beta_{2}$. Solving this system and invoking the results (34)-(35), we end up with

$$
\begin{aligned}
\alpha_{1} & =\frac{\left(k_{1}^{\prime}-k_{4}^{\prime}\right)-\left(k_{2}^{\prime}-k_{5}^{\prime}\right)}{\left(I_{\mu, 1}-I_{\mu, 2}\right)\left(T_{1}-T_{2}\right)} \\
\alpha_{2} & =\frac{I_{\mu, 1}\left(k_{2}^{\prime}-k_{5}^{\prime}\right)-I_{\mu, 2}\left(k_{1}^{\prime}-k_{4}^{\prime}\right)}{\left(I_{\mu, 1}-I_{\mu, 2}\right)\left(T_{1}-T_{2}\right)}, \\
\beta_{1} & =\frac{T_{1}\left(k_{4}^{\prime}-k_{5}^{\prime}\right)-T_{2}\left(k_{1}^{\prime}-k_{2}^{\prime}\right)}{\left(I_{\mu, 1}-I_{\mu, 2}\right)\left(T_{1}-T_{2}\right)} \\
\beta_{2} & =\frac{I_{\mu, 1} T_{1} k_{5}^{\prime}-I_{\mu, 1} T_{2} k_{2}^{\prime}-I_{\mu, 2} T_{1} k_{4}^{\prime}+I_{\mu, 2} T_{2} k_{1}^{\prime}}{\left(I_{\mu, 1}-I_{\mu, 2}\right)\left(T_{1}-T_{2}\right)} .
\end{aligned}
$$

Inserting the parameters (36) into (32), we can deduce the Huggins coefficient as a function of the ionic strength and the temperature.

\subsection{Determination of mobility reduction}

The five above-mentioned series of measurements (8), (11), (22), (28) and (33) allow us to obtain a full description of the intrinsic viscosity and the Huggins coefficient as functions of the ionic strength, the shear rate and the temperature, $[\mu]\left(I_{\mu}, \dot{\gamma}, T\right)$ and $k^{\prime}\left(I_{\mu}, T\right)$. 


\begin{tabular}{|l|c|c|c|c|c|}
\hline \multirow{2}{*}{ Dependence } & Experiments & \multicolumn{2}{|c|}{ Results } & \multicolumn{2}{|c|}{ Parameters } \\
\cline { 2 - 6 } & & {$[\mu]$} & $k^{\prime}$ & for $[\mu]$ & for $k^{\prime}$ \\
\hline \hline \multirow{2}{*}{$\begin{array}{l}\text { Salinity/ } \\
\text { ionic strength }\end{array}$} & $S 1:\left\{\left\{c_{i}\right\}_{i=1}^{N}, I_{\mu, 1}, \dot{\gamma}_{1}, T_{1}\right\}$ & {$[\mu]_{1}$} & $k_{1}^{\prime}$ & $b_{I}$, & $\alpha_{I}$, \\
\cline { 2 - 4 } & $S 2:\left\{\left\{c_{i}\right\}_{i=1}^{N}, I_{\mu, 2}, \dot{\gamma}_{1}, T_{1}\right\}$ & {$[\mu]_{2}$} & $k_{2}^{\prime}$ & {$\left[\mu_{0}\right]$} & $\beta_{I}$ \\
\hline \hline \multirow{2}{*}{ Shear rate } & $S 1:\left\{\left\{c_{i}\right\}_{i=1}^{N}, I_{\mu, 1}, \dot{\gamma}_{1}, T_{1}\right\}$ & {$[\mu]_{1}$} & & $n$ & - \\
\cline { 2 - 4 } & $S 3:\left\{\left\{c_{i}\right\}_{i=1}^{N}, I_{\mu, 1}, \dot{\gamma}_{2}, T_{1}\right\}$ & {$[\mu]_{3}$} & & & \\
\hline \hline \multirow{3}{*}{ Temperature } & $S 1:\left\{\left\{c_{i}\right\}_{i=1}^{N}, I_{\mu, 1}, \dot{\gamma}_{1}, T_{1}\right\}$ & {$[\mu]_{1}$} & $k_{1}^{\prime}$ & $\alpha$ & $\alpha_{T}$, \\
\cline { 2 - 4 } & $S 4:\left\{\left\{c_{i}\right\}_{i=1}^{N}, I_{\mu, 1}, \dot{\gamma}_{1}, T_{2}\right\}$ & {$[\mu]_{4}$} & $k_{4}^{\prime}$ & & $\beta_{T}$ \\
\hline \multirow{2}{*}{$\begin{array}{l}\text { Temperature } \\
+ \text { Salinity }\end{array}$} & $S 2:\left\{\left\{c_{i}\right\}_{i=1}^{N}, I_{\mu, 2}, \dot{\gamma}_{1}, T_{1}\right\}$ & & $k_{2}^{\prime}$ & & $\alpha_{1}, \alpha_{2}$ \\
\cline { 2 - 4 } & $S 5:\left\{\left\{c_{i}\right\}_{i=1}^{N}, I_{\mu, 2}, \dot{\gamma}_{1}, T_{2}\right\}$ & & $k_{5}^{\prime}$ & & $\beta_{1}, \beta_{2}$ \\
\hline
\end{tabular}

Table 1: The summary of the experiments required to determine each of dependence of $[\mu]$ on $I_{\mu}, \dot{\gamma}$, and $T$ and the parameters to deduce for this.

Using the formulations (14), (24) and (30), the complete dependence of the intrinsic viscosity on the ionic strength, the shear rate and the temperature can now be written as

$$
[\mu]\left(I_{\mu}, \dot{\gamma}, T\right)=\frac{\left[[\mu]_{2}+\left([\mu]_{1}-[\mu]_{2}\right) \frac{I_{\mu}^{-1 / 2}-I_{\mu, 2}^{-1 / 2}}{I_{\mu, 1}^{-1 / 2}-I_{\mu, 2}^{-1 / 2}}\right]\left(\frac{[\mu]_{1}}{[\mu]_{4}}\right)^{-\frac{T_{2}\left(T-T_{1}\right)}{T\left(T_{2}-T_{1}\right)}}}{\left[1+\left(\frac{\dot{\gamma}}{\dot{\gamma}_{c}}\right)^{2}\right]^{\frac{\log \left(\frac{[\mu]_{1}}{[\mu]_{3}}\right)}{\log \left(1+\left[\frac{\dot{\gamma}_{2}}{\dot{\gamma}_{c}}\right]^{2}\right)}}} .
$$

As far as the Huggins coefficient is concerned, it becomes equal to

$$
\begin{aligned}
k^{\prime}\left(I_{\mu}, T\right) & =\frac{\left(k_{1}^{\prime}-k_{4}^{\prime}\right)-\left(k_{2}^{\prime}-k_{5}^{\prime}\right)}{\left(I_{\mu, 1}-I_{\mu, 2}\right)\left(T_{1}-T_{2}\right)} I_{\mu} T+\frac{I_{\mu, 1}\left(k_{2}^{\prime}-k_{5}^{\prime}\right)-I_{\mu, 2}\left(k_{1}^{\prime}-k_{4}^{\prime}\right)}{\left(I_{\mu, 1}-I_{\mu, 2}\right)\left(T_{1}-T_{2}\right)} T \\
& +\frac{T_{1}\left(k_{4}^{\prime}-k_{5}^{\prime}\right)-T_{2}\left(k_{1}^{\prime}-k_{2}^{\prime}\right)}{\left(I_{\mu, 1}-I_{\mu, 2}\right)\left(T_{1}-T_{2}\right)} I_{\mu} \\
& +\frac{I_{\mu, 1} T_{1} k_{5}^{\prime}-I_{\mu, 1} T_{2} k_{2}^{\prime}-I_{\mu, 2} T_{1} k_{4}^{\prime}+I_{\mu, 2} T_{2} k_{1}^{\prime}}{\left(I_{\mu, 1}-I_{\mu, 2}\right)\left(T_{1}-T_{2}\right)}
\end{aligned}
$$

Plugging these expressions into formulation (4), the mobility reduction $R_{m}$ can be deduced for any set of parameters $\left\{c, I_{\mu}, \dot{\gamma}, T\right\}$ without performing any new laboratory experiment.

Table 1 summarizes the measurements required to determine the dependence on each parameter. It should be noted that depending on the consid- 
ered case, some measurements may turn out to be useless. For example, if there is no temperature variations in the reservoir, the series of experiments S4 and S5 are not necessary. The intrinsic viscosity and the Huggins coefficient are obtained directly from (24) and (15)-(16), respectively, imposing in the reference set of parameters $T_{1}$ equal to the temperature of the reservoir.

\section{Application to experimental data}

We now apply our calibration methodology to the experimental data set provided by [15], in order to obtain a full reduction mobility description as a function of the salinity (or ionic strength), the shear rate, the temperature and the polymer concentration.

For the experimental data, a hydrolyzed polyacrylamide (HPAM) referenced as FLOPAAM 3630S with molecular weight $M_{w}=20 \times 10^{6}$ daltons is used. The measurements are performed in nano-filtered seawater (NF-SW) of salinity $10 \mathrm{~g} / \mathrm{L}$ and synthetic seawater $(\mathrm{SSW})$ of salinity $33.5 \mathrm{~g} / \mathrm{L}$ at a temperature $T=20^{\circ} \mathrm{C}$.

In [15], the author use viscosimeter to determine rheological properties of polymer. All bulk viscosities were measured using an Antan Paar MCR 301 rheometer. As detailed at the beginning of section 3, it would be better to have polymer viscosity obtained by coreflood but by default, we use these data available in the litterature.

The temperature dependence was not studied in [15]. Therefore, we will use an analytical formula for the viscosity dependence on the temperature [22] and apply it to the measurements of viscosity [15] at low shear rate for various polymer concentrations. Thus, we will obtain the so-called synthetic data that will then be used for the purpose of demonstration of our methodology.

The parameters for five series of measurements (S1-S5) required to determine $R_{m}$ are given in Table 2 .

\subsection{Ionic strength/salinity dependence}

According to the composition of the saline water, NF-SW and SSW, the three groups of ions are determined: two pseudo-cations $\mathrm{I}+, \mathrm{I}++$ and one pseudo-anion I-. Their molar mass $M_{+,++,-}$and mass concentration $C_{+,++,-}$are calculated and inserted into (9) to deduce the corresponding ionic strength $I_{\mu}$. All data are given in Table 3 .

The results of measurements performed with NF-SW by [15] provide the intrinsic viscosity $[\mu]_{1}=6.4 \mathrm{~L} / \mathrm{g}$ and the Huggins coefficient $k_{1}^{\prime}=0.32$ and 


\begin{tabular}{|c|c|c|c|c|}
\hline & $s,[\mathrm{~g} / \mathrm{L}]$ & $\dot{\gamma},\left[\mathrm{s}^{-1}\right]$ & $T,\left[{ }^{\circ} \mathrm{K}\right]$ & $c,[\mathrm{~g} / \mathrm{L}]$ \\
\hline S1 & 10 & 0.1 & 293.15 & $\{0.1,0.25,0.5,0.75,1,1.5,2\}$ \\
\hline S2 & 33.5 & 0.1 & 293.15 & $\{0.1,0.25,0.5,0.75,1,1.5,2\}$ \\
\hline S3 & 10 & 130 & 293.15 & $\{0.1,0.25,0.5,0.75,1,1.5,2\}$ \\
\hline S4 & 10 & 0.1 & 343.15 & $\{0.1,0.25,0.5,0.75,1,1.5,2\}$ \\
\hline S5 & 33.5 & 0.1 & 343.15 & $\{0.1,0.25,0.5,0.75,1,1.5,2\}$ \\
\hline
\end{tabular}

Table 2: Series experiments used to determine $R_{m}\left(c, I_{\mu}, \dot{\gamma}, T\right)$ where $I_{\mu}$ depends on the salinity $s(9)$.

\begin{tabular}{|l|c|c|c||c|c|c||c|c|}
\hline & $M_{+}$ & $M_{++}$ & $M_{-}$ & $C_{+}$ & $C_{++}$ & $C_{-}$ & $s$ & $I_{\mu}$ \\
\hline NF-SW & 23 & 28.17 & 35.75 & 3.85 & 0.05 & 6.10 & 10 & 4.3 \\
\hline SSW & 23.35 & 27.83 & 36.63 & 10.78 & 1.61 & 21.16 & 33.5 & 41.96 \\
\hline
\end{tabular}

Table 3: The saline water characteristics: molar mass $M_{+,++,-}[\mathrm{g} / \mathrm{mol}]$, concentration $C_{+,++,-}[\mathrm{g} / \mathrm{L}]$, salinity $s[\mathrm{~g} / \mathrm{L}]$, ionic strength $I_{\mu}[\mathrm{g} / \mathrm{L}]$.

the measurements with SSW provide $[\mu]_{2}=2.066 \mathrm{~L} / \mathrm{g}$ and $k_{2}^{\prime}=0.35$. Thus, we can calculate $b_{I}$ and $\left[\mu_{0}\right](13)$ and find $[\mu]\left(I_{\mu}\right)$ by $(14)$ as

$$
[\mu]\left(I_{\mu}\right)=13.21 I_{\mu}^{-1 / 2}+0.027 .
$$

If there is no thermal effects, then the Huggins coefficient $k^{\prime}\left(I_{\mu}\right)$ can be deduced from (15) as

$$
k^{\prime}\left(I_{\mu}\right)=0.0008 I_{\mu}+0.32 .
$$

Finally, we compare our correlation with other available experimental data from [15]. For the two salinities (Nf-SW and SSW) and for a temperature $T=20^{\circ} \mathrm{C}$ and a shear stress $\dot{\gamma}=0.1 \mathrm{~s}^{-1}$ we obtain a good agreement between analytical and experimental data as shown in Figure 2.

It should be noted that formulation (4) is done for a low polymer concentration. To adapt (4) for "semi-diluted" regime, we must add a fourth order term. Since this higher order term is not considered in our model, there are light differences for high polymer concentration, especially for NF-SW. However, the data match is pretty good for "diluted regime".

\subsection{Shear rate dependence}

Based on the results obtained by [15] for various shear rates, we could estimate the intrinsic viscosity $[\mu]_{3}=3.75 \mathrm{~L} / \mathrm{g}$. Since we consider that the 


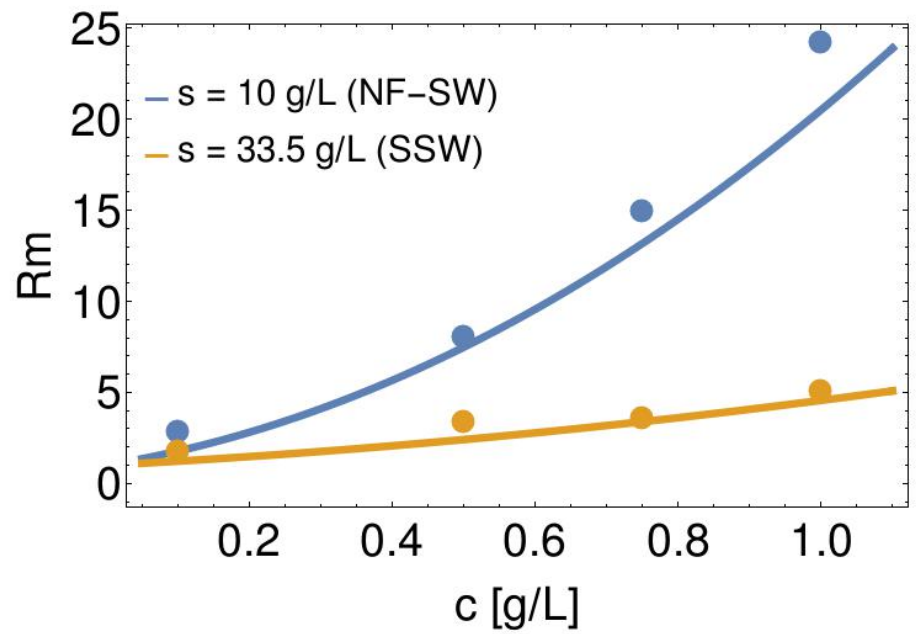

Figure 2: Reduction mobility $R_{m}\left(c, I_{\mu}, \dot{\gamma}=0.1 \mathrm{~s}^{-1}, T=20^{\circ} \mathrm{C}\right)$ function of polymer concentration. The points are experimental data, the plain curve is the correlation presented in this paper.

Huggins coefficient is a constant as function of the shear rate, the estimation of $k_{3}^{\prime}$ is not required.

The characteristic shear rate $\dot{\gamma}_{c}$ is evaluated by (19) with the flow rate $Q=2.88 \times 10^{-4} \mathrm{~m}^{3} / \mathrm{d}$ and the shape factor $a=2.5$ which corresponds to the experimental value obtained for granular media by [5]. Thus, we obtain

$$
\dot{\gamma}_{c}=15.08 \mathrm{~s}^{-1} \text {. }
$$

The power $n$ in (20) is obtained by (23), which results in

$$
n=0.75 \text {. }
$$

Thus, the intrinsic viscosity as a function of the shear rate and the ionic force can be expressed as

$$
[\mu]\left(I_{\mu}, \dot{\gamma}\right)=\frac{[\mu]\left(I_{\mu}\right)}{\left(1+0.0044 \dot{\gamma}^{2}\right)^{0.124}} .
$$

If in the case considered there is no variations of the salinity, then $[\mu]\left(I_{\mu}\right)=$ $[\mu]_{1}$; otherwise, using (39) we obtain

$$
[\mu]\left(I_{\mu}, \dot{\gamma}\right)=\frac{13.21 I_{\mu}^{-1 / 2}+0.027}{\left(1+0.0044 \dot{\gamma}^{2}\right)^{0.124}}
$$




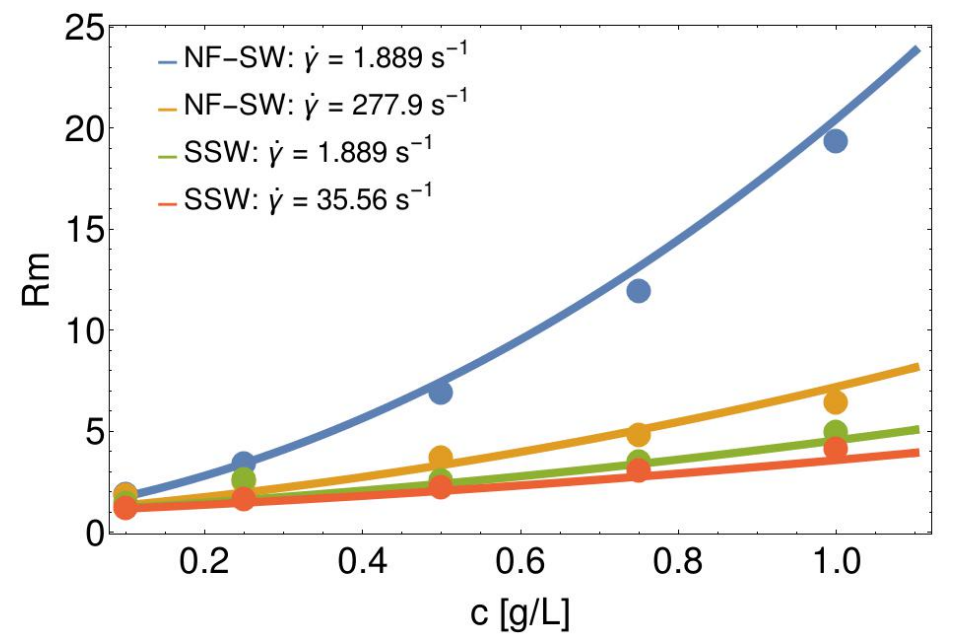

Figure 3: Reduction mobility $R_{m}\left(c, I_{\mu}, \dot{\gamma}, T=20^{\circ} C\right)$ function of polymer concentration for different salinities and different values of shear stress. The points are experimental data, the plain curve is the correlation presented in this paper.

Let us notice that using $I_{\mu}=I_{\mu, 2}=41.96 \mathrm{~g} / \mathrm{L}$ and $\dot{\gamma}=\dot{\gamma}_{1}$, which correspond to series of measurements $\mathrm{S} 2$, the expression (44) yields the intrinsic viscosity $[\mu]\left(I_{\mu, 2}, \dot{\gamma}_{1}\right)=2.066 \mathrm{~g} / \mathrm{L}$. It is in agreement with the intrinsic viscosity obtained directly from the measurements $\mathrm{S} 2,[\mu]_{2}=2.066 \mathrm{~g} / \mathrm{L}$.

Now, we compare our correlation with other available experimental data from [15]. For the two salinities (NF-SW and SSW) and for a temperature $T=20^{\circ} \mathrm{C}$ and different values of shear stress, we obtain a good agreement as shown in Figure 3.

This comparaison shows that the correlation presented in this paper yields the results close to the experimental values.

\subsection{Temperature dependence}

For the temperature dependence of the intrinsic viscosity and the Huggins coefficient, two series of measurements S4 and S5 are required (Table 2). However, we did not find the corresponding measurements for the same polymer and water salinity as used before. In order to demonstrate the relevance of our methodology for the temperature dependencies, we will use the measurements of series $\mathrm{S} 1$ and $\mathrm{S} 2$ for $T_{1}$ and transform them to the data for 
$T_{2}$ using the exponential dependence on $T$ given by [22], i.e.,

$$
\mu(T)=\mu_{0} \exp \left\{\frac{E_{a}}{R}\left(\frac{1}{T}-\frac{1}{T_{1}}\right)\right\}
$$

where $E_{a}=9300 \mathrm{~J} \cdot \mathrm{mol}$ is an activation energy, $R=8.314 \mathrm{~J} \cdot \mathrm{mol}^{-1} \cdot \mathrm{K}^{-1}$ is the ideal gas universal constant and $\mu_{0}$ is the viscosity obtained by [15] for $\dot{\gamma} \rightarrow 0$ for NF-SW (series S1) and SSW (series S2) at temperature $T_{1}$. It should be pointed out that the temperature used in the formulas so far is expressed in ${ }^{\circ} \mathrm{K}$, but for the sake of simplicity, we are switching to the more common unit ${ }^{\circ} \mathrm{C}$.

The viscosity computed by (45) for the temperature $T_{2}=70^{\circ} \mathrm{C}$ for the solvent NF-SW yields the data of series S4, and for the solvent SSW yields the data of series S5. Then, to obtain the intrinsic viscosity by (3), we use $\mu_{w}$ equal to the viscosities measured by [15] for NF-SW and SSW and transformed by (45). Therefore, $[\mu]$ and $k^{\prime}$ are deduced using the linear fit of $\mu\left(T_{2}\right)$ as function of the polymer concentration $c$ (see Section 2). Thus, we obtain the results for series S4 and S5

$$
\begin{array}{ll}
{[\mu]_{4}=3.86 \mathrm{~L} / \mathrm{g},} & k_{4}^{\prime}=0.52, \\
{[\mu]_{5}=1.36 \mathrm{~L} / \mathrm{g},} & k_{5}^{\prime}=0.37 .
\end{array}
$$

Owing to formula (30), the intrinsic viscosity as function of the temperature, shear rate and the salinity/ionic strength is obtained as

$$
[\mu]\left(I_{\mu}, \dot{\gamma}, T\right)=[\mu]\left(I_{\mu}, \dot{\gamma}\right) \exp \left\{6.86\left(\frac{293.15}{T}-1\right)\right\} .
$$

If in the case considered there is no salinity effects, then $[\mu]\left(I_{\mu}, \dot{\gamma}\right)$ is obtained by $(43)$ with $[\mu]\left(I_{\mu}\right)=[\mu]_{1}$. Similarly, if there is no shear rate dependence to account for, then $[\mu]\left(I_{\mu}, \dot{\gamma}\right)$ can be computed by (39). Otherwise, the full description of the intrinsic viscosity $[\mu]\left(I_{\mu}, \dot{\gamma}, T\right)$ is deduced as

$$
[\mu]\left(I_{\mu}, \dot{\gamma}, T\right)=\frac{\left(0.41 I_{\mu}^{-1 / 2}+0.0008\right) \exp \left(\frac{1017.85}{T}\right)}{\left(1+0.0044 \dot{\gamma}^{2}\right)^{0.124}} .
$$

Finally, for the Huggins coefficient $k^{\prime}$ as a function of the salinity and the temperature (32), we obtain

$$
\alpha_{1}=-0.00009, \quad \alpha_{2}=0.0044, \quad \beta_{1}=0.0286, \quad \beta_{2}=-0.959 .
$$




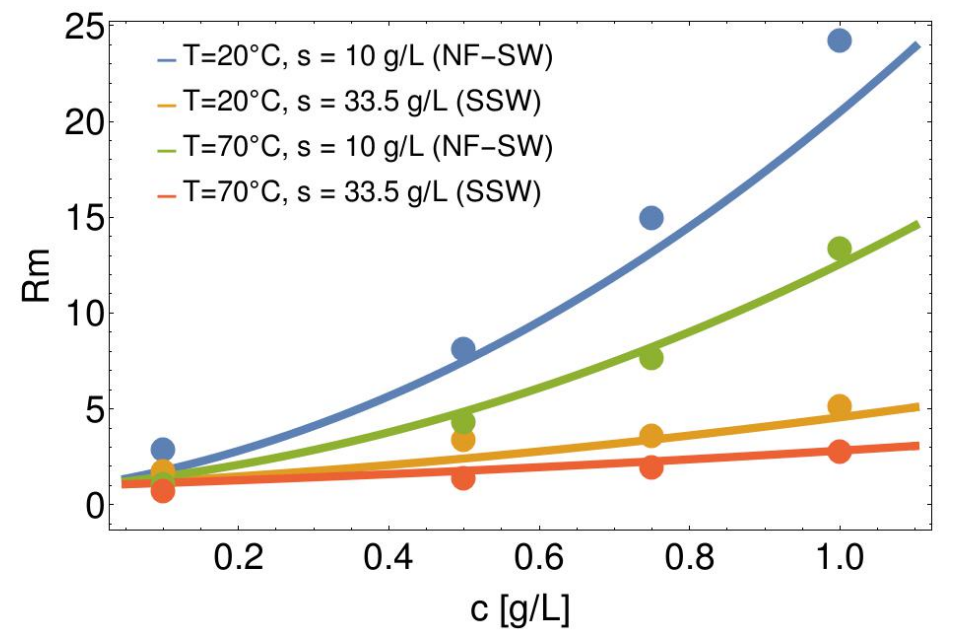

Figure 4: Reduction mobility $R_{m}\left(c, I_{\mu}, \dot{\gamma}=0.1 s^{-} 1, T\right)$ function of polymer concentration for different salinities and different values of temperature. The points at $T=20^{\circ} \mathrm{C}$ are experimental data, the points at $T=70^{\circ} \mathrm{C}$ are synthetic data and the plain curve is the correlation presented in this paper.

Since we assume that $k^{\prime}$ does not depend on the shear rate, the Huggins coefficient $k^{\prime}\left(I_{\mu}, T\right)$ can be written as

$$
k^{\prime}\left(I_{\mu}, T\right)=-0.959+0.0286 I_{\mu}+0.0044 T-0.00009 I_{\mu} T .
$$

Finally, we compare our correlation with experimental data from [15] for a temperature $T=20^{\circ} \mathrm{C}$ and with synthetic datas derived from [15] and obtained by the formula 45. For the two salinities (NF-SW and SSW) and for a shear stress $\dot{\gamma}=0.1 s^{-}$, we obtain a good agreement as shown in Figure 4 .

Let us notice that if there is no salinity dependence, the series S5 is not necessary, and the Huggins coefficient can be deduced by (31) with the coefficients found by (34).

\subsection{Mobility reduction}

The mobility reduction dependence $R_{m}\left(c, I_{\mu}, \dot{\gamma}, T\right)$ on the polymer concentration, the salinity/ionic strength, the shear rate and the temperature is expressed by the Huggins formula (4) using the intrinsic viscosity $[\mu]\left(I_{\mu}, \dot{\gamma}, T\right)$ (49) and the Huggins coefficient $k^{\prime}\left(I_{\mu}, T\right)(51)$. 


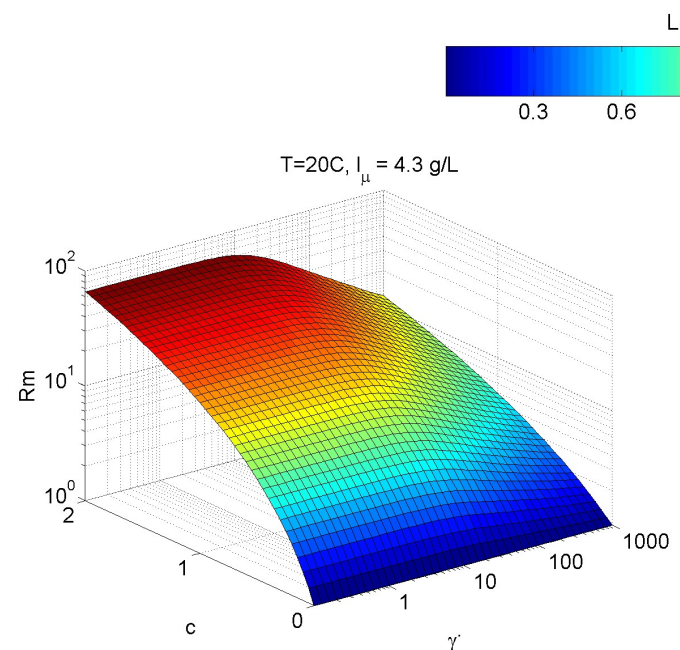

(a)

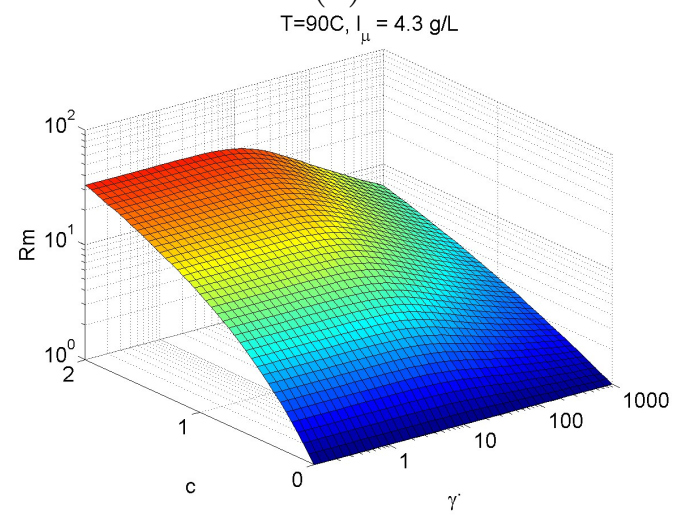

(c)

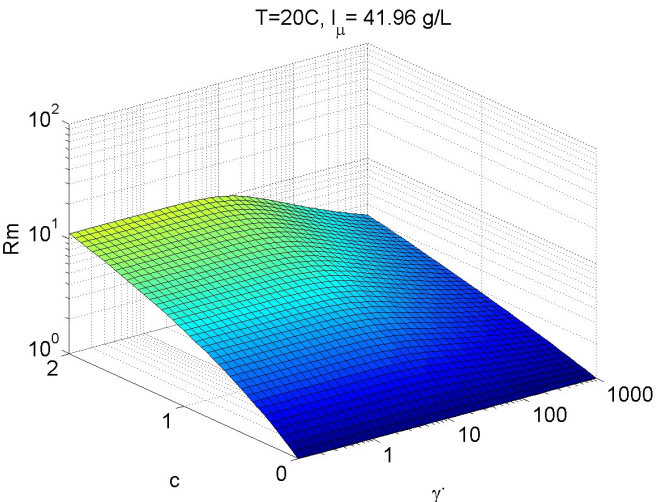

(b)

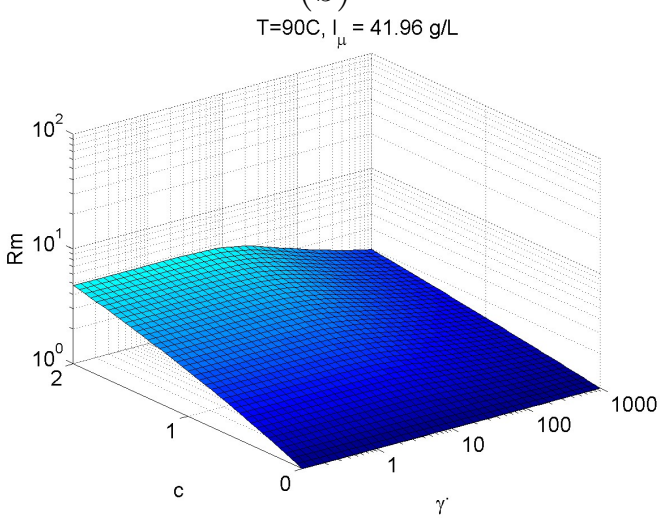

(d)

Figure 5: Reduction mobility $R_{m}\left(c, I_{\mu}, \dot{\gamma}, T\right)$ for $T=20^{\circ} \mathrm{C}(\mathrm{a}, \mathrm{b})$ and $90^{\circ} \mathrm{C}(\mathrm{c}, \mathrm{d})$, and for the ionic force $I_{\mu}=4.3 \mathrm{~g} / \mathrm{L}(\mathrm{a}, \mathrm{c})$ and $41.96 \mathrm{~g} / \mathrm{L}(\mathrm{b}, \mathrm{d})$. 
In Figure 5, $R_{m}\left(c, I_{\mu}, \dot{\gamma}, T\right)$ is shown for $T=20^{\circ} \mathrm{C}$ and $T=90^{\circ} \mathrm{C}$, and for the ionic force $I_{\mu}=4.3 \mathrm{~g} / \mathrm{L}$ and $I_{\mu}=41.96 \mathrm{~g} / \mathrm{L}$. Comparing $R_{m}$ for different values of $T$, it is obvious that the temperature variations play an important role in the mobility reduction and cannot be neglected. For example, increasing the temperature from $20^{\circ}$ to $90^{\circ}$, the mobility reduction factor may decrease up to three times, as illustrated in Figure 5, panels (a) and (c).

Similarly, $I_{\mu}, \dot{\gamma}$ and $c$ have a significant impact on the variations of $R_{m}$. In Figure 5, panels (a) and (b), for instance, increasing the ionic strength from $4.3 \mathrm{~g} / \mathrm{L}$ to $41.96 \mathrm{~g} / \mathrm{L}, R_{m}$ has the effect of decreasing nearly seven times at the most extreme case $\left(c=2 \mathrm{~g} / \mathrm{L}, \dot{\gamma}=1 \mathrm{~s}^{-1}\right)$. Therefore, when dealing with a reservoir that is supposed to have variable temperature, and/or salinity, and/or shear rate and/or polymer concentration, the mobility reduction function should be correctly calibrated according to each presented phenomenon as summarized in Table 1.

\section{Conclusions}

We have presented a new methodology that enables us to completely determine the mobility reduction function $R_{m}$ as a function of the salinity, the temperature and the shear conditions for various polymer concentrations. The most remarkable feature of this determination is that it is achieved by means of a limited number of laboratory experiments, more specifically by using at most five sets of measurements given in Table 1. Application to a literature experimental data set [15] has testified to the relevance of this methodology was testified. This also served to highlight the importance of accounting for each of the mentioned-above dependences in estimating the mobility reduction.

A future work can be to apply this methodology on coreflood experimental results instead of bulk viscosities, with complete thermal results.

\section{Acknowledgements}

The authors would like to thank Quang-Huy Tran for his valuable help and advice in writing this paper.

[1] AlSofi, A.M., Liu, J.S., Han, M., 2013. Numerical simulation of surfactantpolymer coreflooding experiments for carbonates, Journal of Petroleum Science and Engineering, 111, pp.184-196 
[2] AlSofi, A.M., Wang, J. and Kaidar, Z.F., 2018. SmartWater synergy with chemical EOR: Effects on polymer injectivity, retention and acceleration. Journal of Petroleum Science and Engineering, 166, pp.274-282

[3] Amus, T.C., 1982. The Unperturbed Molecular Dimensions of Polyethylene Oxide in Aqueous Solutions from Intrinsic Viscosity Measurements and the Evaluation of the Theta Temperature, Polymer, 23, pp 17751779 .

[4] Carreau, P.J., 1972. Rheological Equations from Molecular Network Theories, J. Rheol., 16, pp 99-127.

[5] Chauveteau, G., 1982. Rodlike Polymer Solution Flow Through Fine Pores: Influence of Pore Size on the Rheological Behavior, J. Rheol., 26(2), pp 111-142.

[6] Cross, M.M., 1964. Rheology of Non-Newtonian Fluids: a New Flow Equation for Pseudoplastic Systems, J. Coll. Sci., 20, pp. 417-437.

[7] Dang, T. Q. C., Chen, Z., Nguyen, T. B. N., Bae, W., Rheological Modeling and Numerical Simulation of HPAM Polymer Viscosity in Porous Media, Journal Energy Sources, Part A: Recovery, Utilization, and Environmental Effects, Volume 37, 2015 - Issue 20

[8] F. Douarche, D. Rousseau, B. Bazin, R. Tabary, P. Moreau and M. Morvan, Modeling Chemical EOR Processes: Some Illustrations from Lab to Reservoir Scale, Oil Gas Sci. Technol. - Rev. IFP Energies nouvelles, 676 (2012) 983-997, DOI: https://doi.org/10.2516/ogst/2012059

[9] Ebaga-Ololo J., Hyun Chon B., Prediction of Polymer Flooding Performance with an Artificial Neural Network: A Two-Polymer-Slug Case, Energies 2017, 10, 844

[10] Huggins, M.L., 1941. Solutions of long chain compounds, Journal of Chemical Physics, 9, p 440.

[11] Lecourtier, J., Rivenq, R., Delaplace, P., Lemonnier, P., Hagry, J. P., Lefevre, D. (1992, January 1). An Innovative Polymer Flooding Simulator Based on Advanced Concepts in Polymer Physics. Society of Petroleum Engineers. doi:10.2118/24150-MS 
[12] P Le Thiez, Physical and thermodynamical aspects in numerical model SARIP, IFP Technical report 40263

[13] Li, Z, Modeling and simulation of polymer flooding including the effects of fracturing, PhD Thesis, University of Texas at Austin, 2015

[14] Medina-Torresa, L., Brito-De La Fuentea, E., Torrestiana-Sanchezb, B., Katthain, R., 2000. Rheological properties of the mucilage gum (Opuntia ficus indica), Food Hydrocolloids 14, pp. 417-424.

[15] Moradi, H., 2011. Experimental Investigation of Polymer Flow Through Water- and Oil-wet Porous Media, MSc, University of Stavanger.

[16] Preux, C., Malinouskaya, I., Nguyen, Q.-L., Tabary, R., 2018. Modeling and Simulating Multi-Polymer Injections, Society of Petroleum Engineers, doi:10.2118/190759-MS

[17] Rodriguez, L., Mejia, A., Reynaud, S., Lespes, G., Favero, C., Antignard S., Giovannetti, B., Gaillard, N., Dupuis, G., Loriau, M., Jouenne, S., Grassl, B., 2016. Monitoring Thermal and Mechanical Stability of Enhanced Oil Recovery EOR Acrylamide Based Polymers PAM Through Intrinsic Viscosity IV Determination Using a New Capillary Rheology Technique, SPE EOR Conference at Oil and Gas West Asia, 21-23 March, Muscat, Oman, doi: 10.2118/179827-MS.

[18] Sharma, A., Delshad, M., Huh, C., \& Pope, G. A. (2011, January 1). A Practical Method to Calculate Polymer Viscosity Accurately in Numerical Reservoir Simulators. Society of Petroleum Engineers. doi:10.2118/147239-MS

[19] Smidsröd, O., Haug, A., 1971. Estimation of Relative Stiffness of the Molecular Chain in Polyelectrolytes from Measurements of Viscosity at Different Ionic Strengths, Biopolymers, 10, pp. 1213-1227.

[20] Sorbie, K.S., 1991. Polymer-Improved Oil Recovery. Springer, Berlin

[21] Torrealba, V.A. and Hoteit, H., 2019. Improved polymer flooding injectivity and displacement by considering compositionally-tuned slugs. Journal of Petroleum Science and Engineering, 178, pp.14-26 
[22] Vinogradov, G.V. and Malkin, A.Y., 1980. Rheology of Polymers, Mir Publishers / Springer-Verlag.

[23] Wolff. C., Viscosité des solutions de polyelectrolytes. Journal de Physique Colloques, 1978, 39 (C2), pp.C2-169-C2-174

[24] Yuan C., Delshad M., Wheeler M.F., Modeling multiphase nonNewtonian polymer flow in IPARS parallel framework, Networks \& Heterogeneous Media, 2010, 5 (3) : 583-602. 